\title{
Optimization of DWDM Demultiplexer Using Regression Analysis
}

\author{
Venkatachalam Rajarajan Balaji, ${ }^{1}$ Mahalingam Murugan, ${ }^{2}$ and Savarimuthu Robinson ${ }^{3}$ \\ ${ }^{1}$ Department of Electronics and Communication Engineering, St. Joseph's Institute of Technology, Chennai, Tamil Nadu 600119, India \\ ${ }^{2}$ Department of Electronics and Communication Engineering, SRM Valliammai Engineering College, Kattankulathur, \\ Tamil Nadu 603203, India \\ ${ }^{3}$ Department of Electronics and Communication Engineering, Mount Zion College of Engineering and Technology, \\ Pudukkottai, Tamil Nadu 622507, India \\ Correspondence should be addressed to Venkatachalam Rajarajan Balaji; vp@valliammai.co.in
}

Received 24 December 2015; Revised 5 May 2016; Accepted 31 May 2016

Academic Editor: James Piao

Copyright ( 2016 Venkatachalam Rajarajan Balaji et al. This is an open access article distributed under the Creative Commons Attribution License, which permits unrestricted use, distribution, and reproduction in any medium, provided the original work is properly cited.

\begin{abstract}
We propose a novel twelve-channel Dense Wavelength Division Multiplexing (DWDM) demultiplexer, using the two-dimensional photonic crystal (2D PC) with square resonant cavity (SRC) of ITU-T G.694.1 standard. The DWDM demultiplexer consists of an input waveguide, SRC, and output waveguide. The SRC in the proposed demultiplexer consists of square resonator and microcavity. The microcavity center rod radius $\left(R_{m}\right)$ is proportional to refractive index. The refractive index property of the rods filters the wavelengths of odd and even channels. The proposed microcavity can filter twelve ITU-T G.694.1 standard wavelengths with $0.2 \mathrm{~nm} / 25 \mathrm{GHz}$ channel spacing between the wavelengths. From the simulation, we optimize the rod radius and wavelength with linear regression analysis. From the regression analysis, we can achieve $95 \%$ of accuracy with an average quality factor of 7890 , the uniform spectral line-width of $0.2 \mathrm{~nm}$, the transmission efficiency of $90 \%$, crosstalk of $-42 \mathrm{~dB}$, and footprint of about $784 \mu \mathrm{m}^{2}$.
\end{abstract}

\section{Introduction}

Fiber cable transfers the data for the long distance without loss, when compared to the coaxial transmission. However, individual fiber connectivity to single consumer needs robust and expensive routing system. To overcome the high-priced routing SMF connectivity to each consumer is the solution.

The single-mode optical fiber (SMF) works with wavelength division multiplexing (WDM) and DWDM techniques, for transferring multiple light waves with precise wavelengths inside the fiber. The SMF DWDM system in demultiplexer at the consumer end separates the multiple light wavelengths [1]. The demultiplexers are classified as passive and active demultiplexers. The passive demultiplexers design consists of frequency filters [2], prisms [3], and diffraction gratings [2]. The active demultiplexer comprises passive components and tunable detectors [4]. The disadvantages of active and passive demultiplexers are as follows: low normalized transmission power, high crosstalk, low-quality factor, and scale of centimetres. The disadvantages of demultiplexers are overcome by replacing them with Photonics Integrated Circuits (PICs).

PICs consist of photonic crystals (PCs) which can maintain hundreds of channels through micro dimension scale. The growth of PCs in PIC drastically increases in the field of fiber optics. Photonic crystals are low loss periodic dielectric electromagnetic medium, with Photonic Band Gaps (PBGs). The PCs control the propagation of light with PBG. The PBG structure does not allow the light with different wavelengths in any direction $[5,6]$. By introducing defects in the periodic structures the PBG is entirely broken which allows the different wavelength inside the structure. Typically, there are two kinds of defects reported, namely, line defects and point defects. The removal/change of structural parameters of the rods in a row or column is denoted as line defect; however, the change/removal of structural parameters of single rods are termed point defects. The structural parameters are the radius of the rod, the refractive index, and the lattice constant. 
The line and point defects are very important to realize PC based optical devices. The PCs with defects can apply in designing various devices such as wavelength demultiplexers [7], beam splitters [8], optical logical encoder [9], ring resonators [10], and photonic sensors [11].

In WDM wavelength demultiplexers play a vital role for point-to-point networks, which are classified as coarse wavelength division multiplexing $(\mathrm{CWDM})$ and DWDM. The CWDM comprises the ITU-T G.694.2 standard of eight wavelengths with $20 \mathrm{~nm}$ channel spacing. The DWDM consists of ITU-T G.694.1 criterion of 0.1, 0.2, 0.4, and $0.8 \mathrm{~nm}$ channel dispersing with 512 distinct wavelengths. The channel-dispersing feature of the DWDM standard attracts many researchers to improve the DWDM criterion. However, DWDM technology in fiber optics provides low attenuation and the dynamic usage of bandwidth for SMF. The SMF with DWDM technology uses multiple wavelengths as carriers and concedes them to transmit in the fiber concurrently to the consumer end.

From the literature survey, it is deemed that the 2D PC based demultiplexer for CWDM and the DWDM system is reported using line defects [12-17], ring resonant cavity [18, 19], and so forth, for introducing the line and point defects. As the ITU-T G.694.1 DWDM system has many benefits, which are very relevant, the authors have considered the DWDM system and presented the research results here. The DWDM demultiplexer using 2D PC has a T-shaped structure with line defects resonant cavity [12-14] and P-shaped single resonant cavity with different rod radius to drop different wavelengths [20] and, hence, the multi-T-shaped structure with line/point defects [15-17], X-ring cavity [18], and resonant cavity/line defects [19]. From the literature survey, it is identified that the demultiplexer is designed using different shapes of the cavity. However, the transmission efficiency, crosstalk, and $Q$ factor need improvement to meet the future requirements. Hence, in this paper, a new square ring resonant cavity/point defects based demultiplexer has been proposed and designed to work with constructive interferences that result in high transmission efficiency.

The DWDM demultiplexer is of two and four channels. The drawbacks of the earlier works are less channel number, nonuniform spectral line-width for dropped channels, low $Q$ factor, and low transmission efficiency [12-19]. The DWDM of six and eight channels to improve the aforementioned parameters with novel demultiplexer structure is formed. The increasing channels in DWDM reduce the cost, and best optimization is obtained since the number of users increased with the number of channels. The proposed 12channel DWDM has odd and even distinct color wavelengths. The demultiplexer is formed with the combination of square ring resonator, a waveguide, and microcavity rods in a $2 \mathrm{D}$ photonic crystal. In the demultiplexer for developing 12channel DWDM, we design a filter in a position to drop highintensity resonant wavelength on the influence of microcavity inner $\operatorname{rod}\left(R_{m}\right)$. The radius of the $\operatorname{rod}\left(R_{m}\right)$ is $100 \%$ reflection situation.

This paper is prepared as follows: Section 2 discusses the band diagram for $50 * 50 \mathrm{PC}$ structures earlier than inducing the defects. Section 3 describes the design of microcavity and

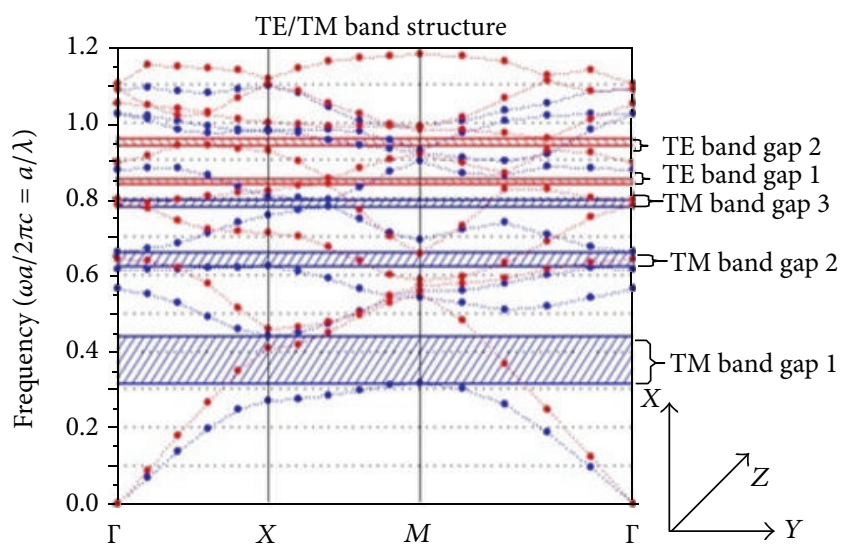

FIgURE 1: Band diagram for $50 * 50$ PC structures before inducing the defects.

its features. The novel design for 12-channel demultiplexer is proposed in Section 4. The simulations outcome and discussion are analyzed in Section 5. Section 6 discusses the regression evaluation to optimize the radius of rods with wavelength. Subsequently, Section 7 concludes the paper.

\section{Photonic Crystal Geometry}

The proposed DWDM demultiplexer uses the 2-dimensional photonic crystal square lattice, and it will be better to attain horizontal confinement of light by the use of square lattice structure. The filter is developed with $50 \times 50$ rods in the $X$ and $Z$ direction for higher coupling of modes. The high refractive index Si rods and background air are 3.48 and 1; each rod has a radius $R=110 \mathrm{~nm}$, where the lattice constant $a=560 \mathrm{~nm}$ and silicon permittivity $(\varepsilon)=11.9$.

The analysis of PC band structure is studied by the Plane Wave Expansion (PWE) method. The PWE method calculates PBG in propagation modes for the periodic and nonperiodic structures [21]. The electromagnetic wave propagation modes in photonic crystal analysis follow Maxwell's equations:

$$
\nabla \times\left(\frac{1}{\varepsilon(r)} \nabla \times E(r)\right)=\frac{\omega^{2}}{c^{2}} E(r),
$$

where $\varepsilon(r)$ represents the dielectric function, " $\omega$ " is the angular frequency, $E(r)$ represents the electric field of periodic structure, and " $c$ " is the speed of the light. From (1), the 2D PC band structure is determined.

The photonic crystal realizes the fields in two polarizations: TE polarization where the electrical field is polarized in $X Y$ plane, that is, magnetic field in direction of propagation $(Z)$, and TM polarization, the place the magnetic field is parallel to the interface and electric field is within the propagation direction $(Z)$.

The proposed band structure before introducing the defects is shown in Figure 1, which has three TM PBGs and two TE PBGs in the band diagram as shown in Figure 1. The normalized frequency and its wavelength range are listed in Table 1. In the table, first TM PBG wavelength lies between 


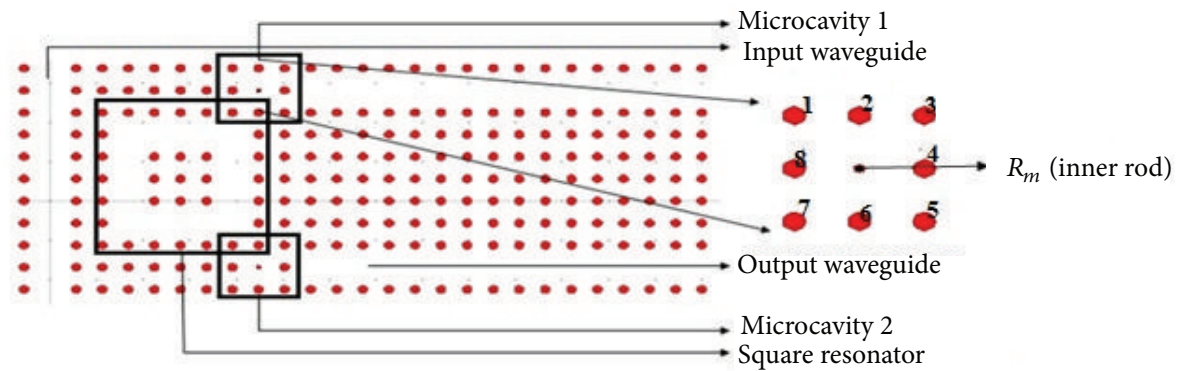

FIGURE 2: A single resonant cavity with two microcavities.

TABLE 1: Photonic band gap and normalized frequency and its corresponding wavelength of the proposed structure.

\begin{tabular}{lccc}
\hline $\begin{array}{l}\text { S. } \\
\text { number }\end{array}$ & PBG & Normalized frequency $(a / \lambda)$ & Wavelength $(\mathrm{nm})$ \\
\hline \multirow{3}{*}{1} & \multirow{2}{*}{ TM PBG } & $0.31-0.445$ & $1258-1806$ \\
& & $0.588-0.6455$ & $867-952$ \\
& $0.768-0.778$ & $729-719$ \\
\hline \multirow{2}{*}{ TE PBG } & $0.923-0.934$ & $599-606$ \\
& & $0.825-0.835$ & $670-678$ \\
\hline
\end{tabular}

$1258 \mathrm{~nm}$ and $1806 \mathrm{~nm}$ for low loss communication, since the frequency lies in the third window of optical communication in proposed work. All the simulation performs with TM mode where the electric field is perpendicular to the rod axis. The proposed band structure design uses a 12-channel DWDM filter. Finite Difference Time Domain (FDTD) is utilized to analyze the transmission and reflection spectrum of the photonic device including photonic crystals. The FDTD method simulates the propagation of electromagnetic waves inside the PCs [22].

\section{Microcavity Design}

Figure 2 shows the single resonant cavity with two microcavities. The microcavity with point defect shown is separated and magnified, in Figure 2. The microcavity with point defects is introduced. The point defect is created by altering the shape, size, and dielectric constant of the rods. The sizes of the rods increase with the change of refractive index; so, the desired wavelength is tuned. The SRC has a square resonator and microcavity. The microcavity consists of one center rod surrounded by 8 reflecting rods. The 8 rods are used to control the width of the resonant frequency in the band gap. The center rod $\left(R_{m}\right)$ is used to tune the desired wavelength with high intensity. The square resonator is used to localize the photon inside the region. Once the distinct photons couple with the microcavity, it tunes the coupled wavelength with the high resonant frequency with the presence of the rod $\left(R_{m}\right)$. The high resonant frequency for $1554.9 \mathrm{~nm}$ is shown in Figure 3 after simulation.

The microcavity performance is analyzed with $Q$ factor. Typically, the $Q$ factor determines the selectivity of the demultiplexer and the $Q$ factor is calculated separately for all the channels using the following formula:

$$
Q \equiv \omega_{O} \tau_{p h} \equiv \frac{\lambda_{r}}{\Delta \lambda}
$$

where $\lambda_{r}$ is resonant wavelength of the desired channel and $\Delta \lambda$ represents the Full Width at Half Maximum point for the desired channel. In the proposed DWDM model, the microcavity increases the $Q$ factor and shows better performance when compared to the existing DWDM demultiplexer.

\section{Multichannel Filter Design}

Figure 4 shows the twelve-channel DWDM demultiplexer schematic. The proposed DWDM demultiplexer utilizes $50 \times$ 50 rods, with the $784 \mu \mathrm{m}^{2}$ lattice structure, which can acquire narrower spectral line-width. The DWDM also filters precise color wavelength through the constitution of the resonant cavity. The square resonant cavity filter consists of square ring resonator and microcavity rods. The square ring resonator straps the photons in the cavity when low resistance with specific color wavelength occurs and the color wavelength couples to microcavity cells. In this novel design, microcavity cells work as wavelength selective filter to drop the desired wavelengths. The rods in microcavity cells work to separate the even and odd color wavelengths by adjusting the radius of microcavity inner rods $\left(R_{m}\right)$. The radius of the microcavity interior rod $\left(R_{m}\right)$ increases due to the high-intensity resonant wavelength. As soon as the radius of the $R_{m}$ increases, the refractive index in rods increases due to the fact that the resonant wavelength also shifts with $0.2 \mathrm{~nm}$ channel spacing. The rods around the microcavity control the width of the channel spacing of desired color wavelengths. Figure 2 shows the base of the single resonant cavity designed to 12-channel DWDM demultiplexer. The 12-channel DWDM demultiplexer design has six resonant cavities, where each cavity is to drop two distinct color wavelengths. The six resonant cavities are positioned between the input bus waveguide and the output drop waveguide. The input bus waveguide is designed by eliminating 47 rods in the lattice. Likewise output drop waveguide works with removing 16 rods in each channel. The 15 rods are left for designing the square resonant cavity. The distance between waveguide and the cavity is deemed to be 


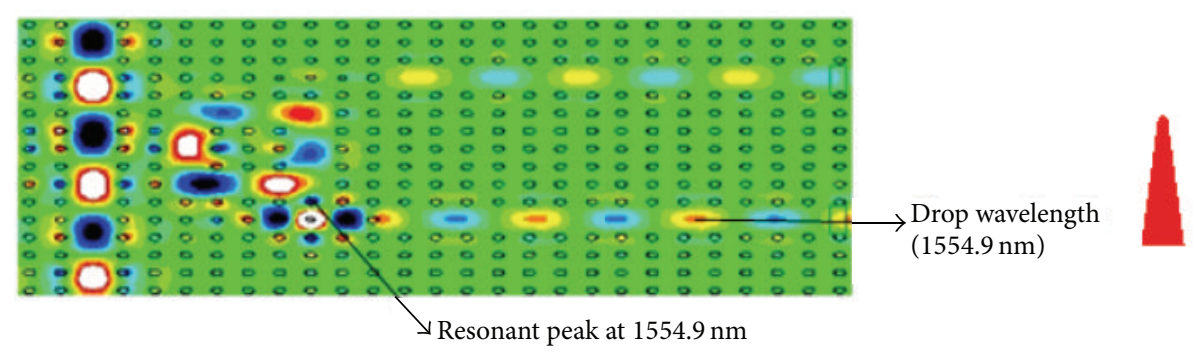

FIGURE 3: Electric field distribution of a single resonant cavity for color wavelength $1554.9 \mathrm{~nm}$.

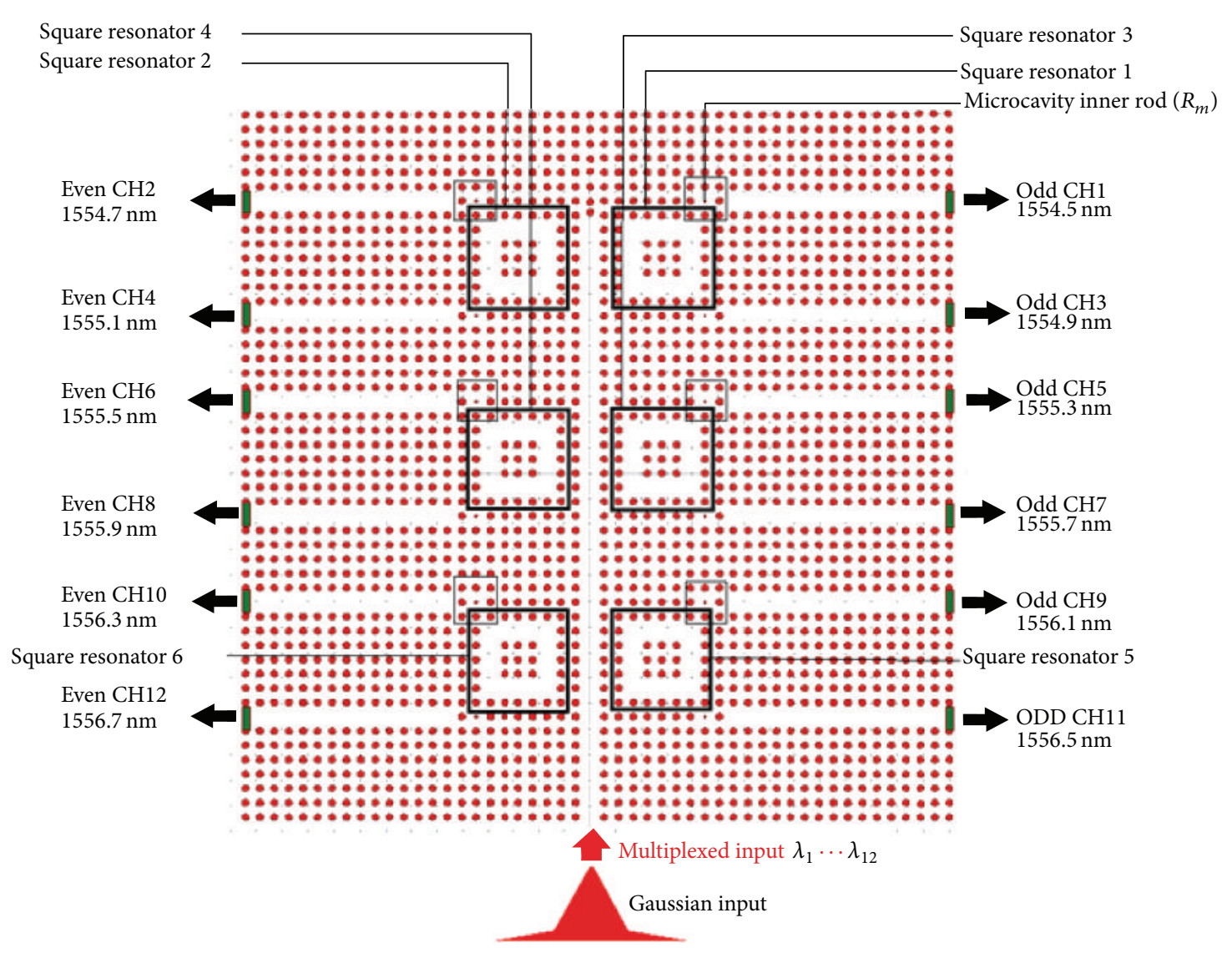

FIGURE 4: Schematic diagram of the proposed twelve-channel demultiplexer using PC square resonant cavity.

good with two rows of rods in order to reduce the coupling loss and the back reflection loss.

The novel resonant cavity works with two microcavities, one microcavity positioned at the higher end of the cavity and the other microcavity placed below the cavity. The microcavity is partitioned into eight rods and core rod radius named $R_{m}$. The radius of rods $\left(R_{m}\right)$ increases for every microcavity to filter the desired color wavelength.

The walls of the cavity are used to control the width of a resonant frequency. To design a 12-channel DWDM demultiplexer, we include six resonant cavities. Each cavity design has two branches with distinct defects in its dimension. The two channel branches from the single resonant cavity with a separation of seven rows of rods are shown in Figure 4. The proposed design exhibits more quantity of channels with the small footprint. As we stated previously with increasing microcavity core rods $\left(R_{m}\right)$ are able to shift the resonant wavelength on the higher side which controls the channel spacing $(0.2 \mathrm{~nm})$ through the surrounding rods of the microcavity. We added point defects $\left(R_{m}\right)$ with 12 different sizes to drop 12 channels. The radius $\left(R_{m}, m=\right.$ $1,2,3,4,5,6,7,8,9,10,11,12)$ is given as $R_{1}=41.5 \mathrm{~nm}, R_{2}=$ $41.95 \mathrm{~nm}, R_{3}=42.4 \mathrm{~nm}, R_{4}=42.85 \mathrm{~nm}, R_{5}=43.3 \mathrm{~nm}, R_{6}=$ $43.75 \mathrm{~nm}, R_{7}=44.20 \mathrm{~nm}, R_{8}=44.65 \mathrm{~nm}, R_{9}=45.10 \mathrm{~nm}$, $R_{10}=45.55 \mathrm{~nm}, R_{11}=46 \mathrm{~nm}$, and $R_{12}=46.45 \mathrm{~nm}$.

The desired wavelengths are the drop with a radius of the microcavity inner rods $\left(R_{m}\right)$ due to the filter. Other parameters are constant such as nondefected rods, lattice constant, and refractive index. The channels are designed to drop the desired wavelength with high transmission efficiency, 


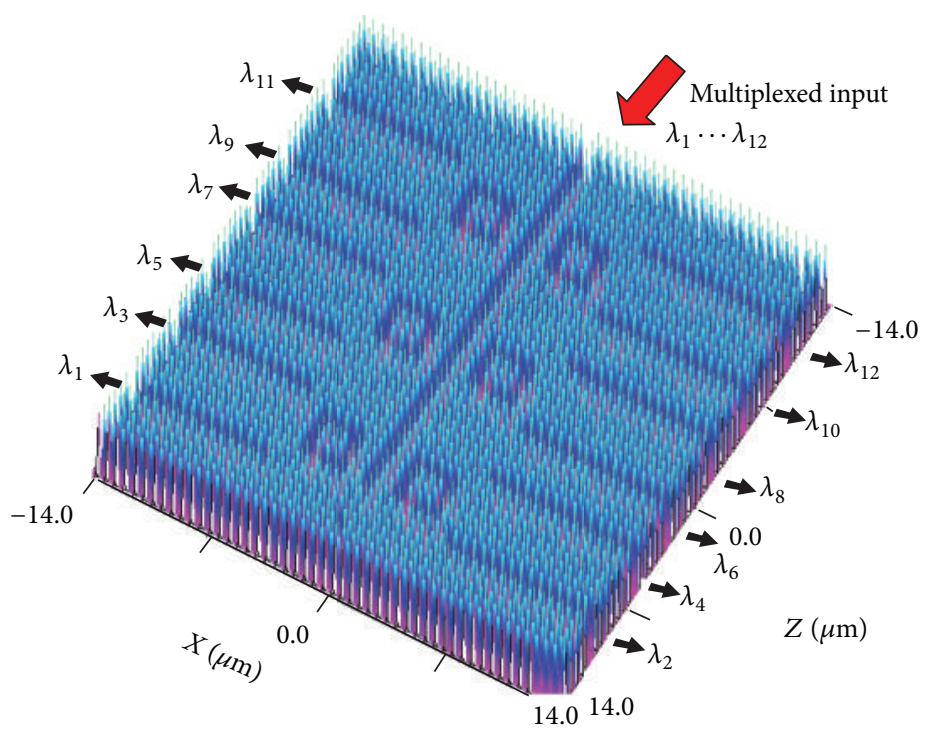

FIGURE 5: 3D view of the proposed twelve-channel demultiplexer.

high $Q$, and narrow line-width. The designed demultiplexer selects the radius of the microcavity inner rods $\left(R_{m}\right)$ with optimized simulation under different conditions, like characteristics of rods, the lattice constant, and refractive index. The demultiplexer is designed with twelve ports to drop the twelve desired wavelengths. The 3D view of proposed twelvechannel demultiplexer is depicted in Figure 5. The size of the demultiplexer is $28 \mu \mathrm{m} \times 28 \mu \mathrm{m}$, which is very small; hence, it could be easily deployed in PICs.

\section{Simulation Results and Discussion}

The proposed DWDM demultiplexer is simulated using FDTD with PML ABC. The proposed crystal structure utilizes PML ABC (PML Absorbing Boundary Conditions) to optimize the boundary region without reflection of electromagnetic waves. To observe boundary conditions without any reflection of electromagnetic waves for all the frequency and angle of incidence with PML layer, formulate Maxwell's equations [23].

The absorption rate is higher in the PML ABC structure, whereas other boundary conditions show more reflections during simulations. The proposed model utilizes PML width and reflection as $500 \mathrm{~nm}, \mathrm{PML}$, and $10^{-8}$, respectively. The FDTD grid size in the simulation at $28 \mathrm{~nm}(x / 20=0.05 a)$ works significantly in DWDM environment. The proposed 12-channel DWDM demultiplexer makes use of spatial Gaussian pulse within the input waveguide. The power monitor is placed on the end of the waveguide with the intention of receiving the normalized output transmission. The output transmission spectrum is acquired using Fourier transform of the electric field using the monitor. The normalized transmission is calculated with

$$
T(f)=\frac{1 / 2 \int \operatorname{real}\left(p(f)^{\text {monitor }}\right) \cdot d S}{\text { SourcePower }},
$$

where $T(f)$ denotes normalized transmission about the frequency, $p(f)$ denotes the pointing vector, and $d S$ denotes surface normal preserving right time step in the FDTD simulation having an effect on DWDM environment. The time step should follow this rule in the filter:

$$
\Delta t \leq \frac{1}{c \sqrt{1 / \Delta X^{2}+1 / \Delta Y^{2}}}
$$

where $\Delta t$ represents the step time and $c$ represents the speed of light in free space. The filter is simulated with an increment of $0.0001 \mathrm{~nm}$ for a $4476 \mathrm{~min}$ runtime for memory structure of 53.6 MB to get high $Q$ factor output. The size of the structure in $X$ and $Z$ direction is $28 \mu \mathrm{m}$ and $28 \mu \mathrm{m}$, so the footprint proposed demultiplexer is $784 \mu \mathrm{m}^{2}$.

In the previous work on the DWDM demultiplexer, more focus was on four channels and less focus was on eight channels. The increasing channels in DWDM reduce the cost, and the best optimization is obtained since number of users increased with number of channels. The paper shows the results with 12-channel DWDM demultiplexer. The proposed design utilized the benefits of both square resonator and novel microcavity. The square resonator straps the modes within the cavity with the sustained time and microcavity tuned to couple the distinct color wavelength with high resonant frequency simply with point defects. The resonant cavity is designed with the novel microcavity. The novel microcavity can filter the distinct DWDM wavelengths with the change in inner rods. The radius of inner rods is optimized for simulation under different conditions (characteristics of rods, a lattice constant, and refractive index) to separate odd and even distinct wavelengths. This approach drastically reduces the crosstalk between neighbour channels in practical.

The output spectra of proposed twelve-channel demultiplexer for odd channels and even channels are as shown in Figures 6 and 7. The wavelengths in Figures 6, 7, and 8 attain 
TABLE 2: The summary of various parameters of the twelve-channel DWDM demultiplexer for odd channels.

\begin{tabular}{|c|c|c|c|c|}
\hline $\begin{array}{l}\text { DWDM } \\
\text { channel/wavelength } \\
\lambda_{r}(\mathrm{~nm}) \\
\end{array}$ & Defect rod $\left(R_{m}\right)(\mathrm{nm})$ & Spectral linewidth $(\Delta \lambda)$ in $(\mathrm{nm})$ & $Q$ factor & Efficiency (\%) \\
\hline$\lambda_{1}-1554.5 \mathrm{~nm}$ & 41.5 & 0.2 & 7772.5 & 90 \\
\hline$\lambda_{3}-1554.9 \mathrm{~nm}$ & 42.4 & 0.2 & 7774.5 & 94 \\
\hline$\lambda_{5}-1555.3 \mathrm{~nm}$ & 43.3 & 0.2 & 7776.5 & 98 \\
\hline$\lambda_{7}-1555.7 \mathrm{~nm}$ & 44.20 & 0.2 & 7778.5 & 93 \\
\hline$\lambda_{9}-1556.1 \mathrm{~nm}$ & 45.10 & 0.2 & 7780.5 & 99 \\
\hline$\lambda_{11}-1556.5 \mathrm{~nm}$ & 46 & 0.2 & 7782.5 & 92 \\
\hline
\end{tabular}

TABLE 3: The summary of various parameters of the twelve-channel DWDM demultiplexer for even channels.

\begin{tabular}{|c|c|c|c|c|}
\hline $\begin{array}{l}\text { DWDM } \\
\text { channel/wavelength } \\
\lambda_{r}(\mathrm{~nm}) \\
\end{array}$ & Defect $\operatorname{rod}\left(R_{m}\right)(\mathrm{nm})$ & Spectral linewidth $(\Delta \lambda)$ in $(\mathrm{nm})$ & $Q$ factor & Efficiency (\%) \\
\hline$\lambda_{2}-1554.7 \mathrm{~nm}$ & 41.95 & 0.2 & 7773.5 & 99.4 \\
\hline$\lambda_{4}-1555.1 \mathrm{~nm}$ & 42.85 & 0.2 & 7775.5 & 95.3 \\
\hline$\lambda_{6}-1555.5 \mathrm{~nm}$ & 43.75 & 0.2 & 7777.5 & 100 \\
\hline$\lambda_{8}-1555.9 \mathrm{~nm}$ & 44.65 & 0.2 & 7779.5 & 94 \\
\hline$\lambda_{10}-1556.3 \mathrm{~nm}$ & 45.55 & 0.2 & 7781.5 & 91.3 \\
\hline$\lambda_{12}-1556.7 \mathrm{~nm}$ & 46.45 & 0.2 & 7783.5 & 99 \\
\hline
\end{tabular}

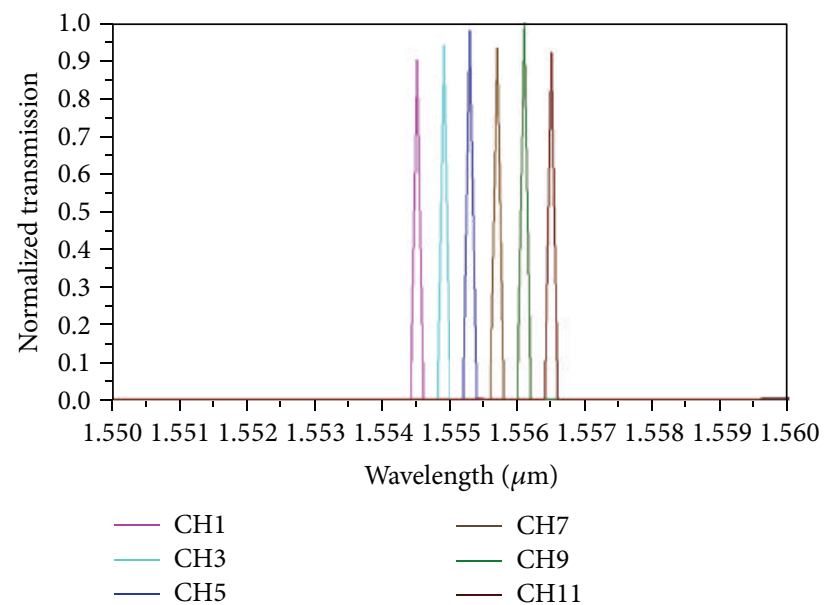

FIGURE 6: Normalized output spectra of twelve-channel DWDM demultiplexer for the odd channel.

ITU-T G.694.1 DWDM system and the output spectra of twelve-channel DWDM demultiplexer are shown in Figure 8.

The resonant wavelengths of the DWDM system observed at $1554.5 \mathrm{~nm}, 1554.9 \mathrm{~nm}, 1555.3 \mathrm{~nm}, 1555.7 \mathrm{~nm}$, $1556.1 \mathrm{~nm}, 1556.5 \mathrm{~nm}, 1554.7 \mathrm{~nm}, 1555.1 \mathrm{~nm}, 1555.5 \mathrm{~nm}$, $1555.9 \mathrm{~nm}, 1556.3 \mathrm{~nm}$, and $1556.7 \mathrm{~nm}$ lie in $C$ band of the optical window. The $C$ band window widely prefers the network due to low loss communication. The complete specifications of the twelve-channel DWDM demultiplexer for odd and even channels are listed in Tables 2 and 3.

In this paper, authors designed 2D PC based 12-channel demultiplexer for DWDM ITU-T G.694.1 standard system.

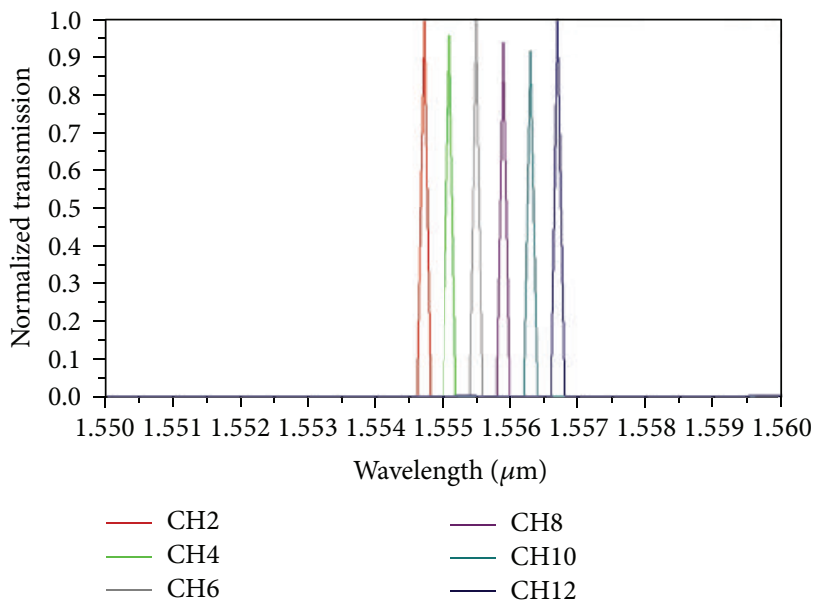

FIGURE 7: Normalized output spectra of twelve-channel DWDM demultiplexer for even channel.

Hence we have considered that the wavelength range lies between $1554.5 \mathrm{~nm}$ and $1556.7 \mathrm{~nm}$ with $0.2 \mathrm{~nm}$ channel spacing. In order to attain the normalized output spectra which are shown in Figures 6-9, authors considered the wavelength increment (resolution) as $0.0001 \mathrm{~nm}$ from $1550 \mathrm{~nm}$ to $1560 \mathrm{~nm}$ (i.e., the total simulation period (time) is about 4476 minutes). The quality factor is calculated from (2); for example, the resonant wavelength and Full Width at Half Maximum of channel 1 are $1554.5 \mathrm{~nm}$ and $0.2 \mathrm{~nm}$, respectively. From the above value, authors attained the $Q$ factor value of 7772.5. Similarly, the $Q$ factors calculated for all the other channels are listed in Tables 2 and 3. 


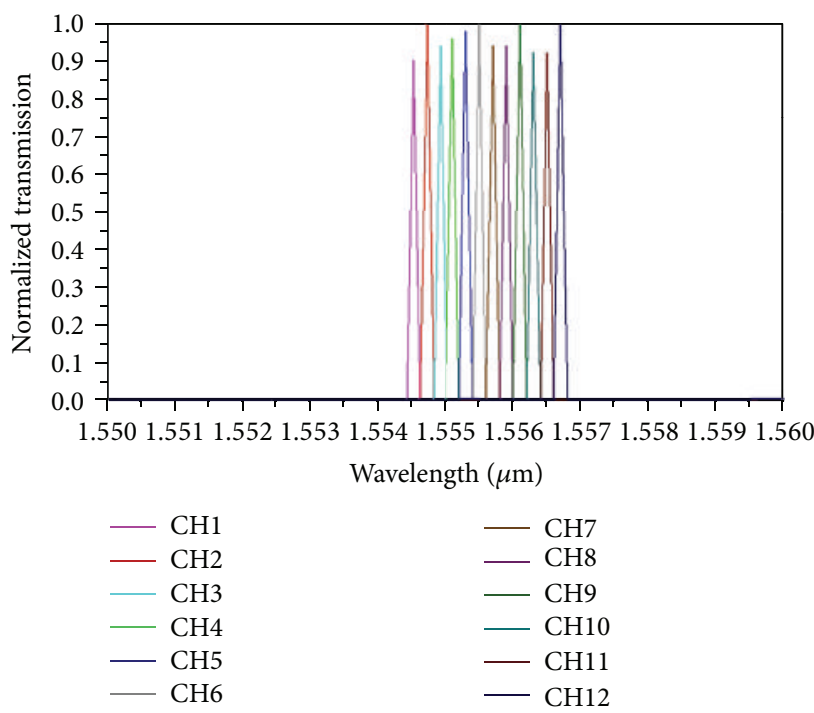

FIGURE 8: Normalized output spectra of twelve-channel DWDM demultiplexer with uniform spectral line-width $0.2 \mathrm{~nm}$.

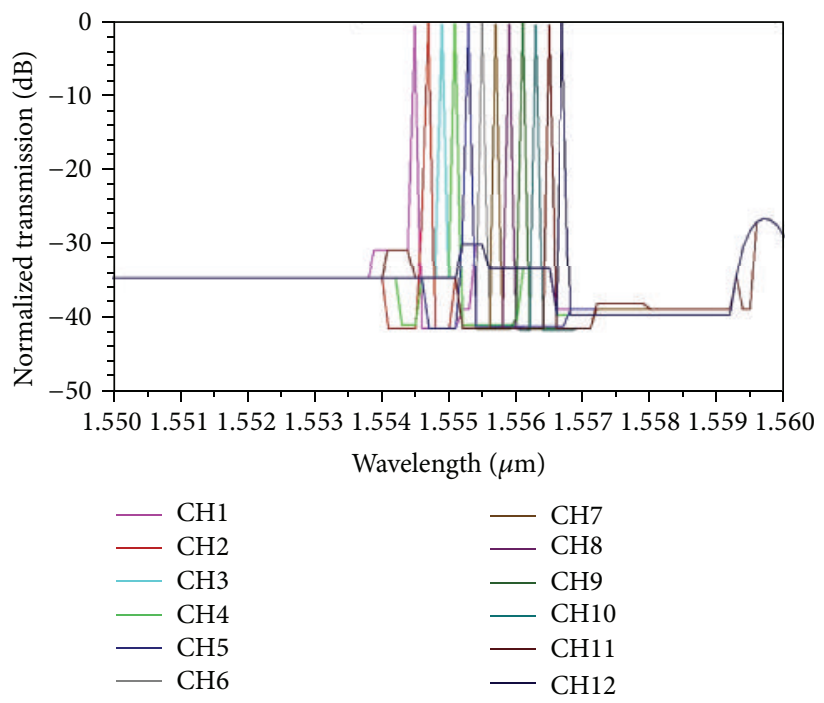

FIGURE 9: The spectral output of the twelve-channel DWDM demultiplexer in dB scale.

There are several microfabrication methods available for fabricating 2D PC. They are optical UV lithography, direct UV laser writing, electron-beam lithography, focused ion beam, and multiexposure holography. Above all, electronbeam lithography and dry-etching techniques offer remarkable resolution of fabrication of PC based devices [24, 25]. In addition, technology could support fabricating the device with $\mathrm{nm}$ resolution. In addition, there are several attempts already made with less than $0.45 \mathrm{~nm}$ [14-17]. Hence our proposed work could be realized in near future.

The biggest challenge in designing DWDM demultiplexer is to obtain low crosstalk. The demultiplexer design is the focus to improve low crosstalk with proposed SRC cavity. The spectral response of demultiplexer in $\mathrm{dB}$ scale is seen in Figure 9 and is used to calculate the crosstalk among the channels $\left(C_{i j}\right)$. From Figure 9, we observe that the crosstalk of the channels varied over the range from $-30 \mathrm{~dB}$ to $-42 \mathrm{~dB}$, which produces much small crosstalk, compared to the previous results. The crosstalk between channels is $C_{i j}$, where $i$ and $j$ denote the channel number. For example, $C_{23}$ gives the crosstalk between channel 2 and channel 3. The crosstalk among the channels is listed in Table 4.

The functional characteristics of the proposed twelvechannel DWDM demultiplexer are compared to the reported DWDM demultiplexers, which are listed in Table 5. From Table 5, it is proved that the proposed square resonant cavity based demultiplexer works better than the existing DWDM design. From the simulation of various wavelengths, we achieved functional parameters such as a quality factor of 8000 , the spectral line-width of $0.2 \mathrm{~nm}$, a transmission efficiency of $90-100 \%$, crosstalk of $-42 \mathrm{~dB}$, and device size of about $784 \mu \mathrm{m}^{2}$. From the results, the existing DWDM system 
TABLE 4: Crosstalk values $\left(C_{i j}\right)$ of proposed twelve-channel PC based demultiplexer $(\mathrm{dB})$.

\begin{tabular}{lcccccccccccc}
\hline Channels $\left(C_{i j}\right)$ & $\lambda_{1}$ & $\lambda_{2}$ & $\lambda_{3}$ & $\lambda_{4}$ & $\lambda_{5}$ & $\lambda_{6}$ & $\lambda_{7}$ & $\lambda_{8}$ & $\lambda_{9}$ & $\lambda_{10}$ & $\lambda_{11}$ & $\lambda_{12}$ \\
\hline$\lambda_{1}$ & NA & -30.7 & -41.5 & -41.5 & -39.1 & -30.1 & -33.2 & -33.4 & -33.4 & -33.4 & -33.3 & -39 \\
$\lambda_{2}$ & -31 & NA & -32 & -37 & -30 & -30 & -33.3 & -33.4 & -33.2 & -33.3 & -33.4 & -39.6 \\
$\lambda_{3}$ & -33 & -34.2 & NA & -31 & -41.6 & -41.6 & -41.6 & -41.6 & -41.6 & -41.6 & -41.6 & -41.6 \\
$\lambda_{4}$ & -34 & -34 & -31 & NA & -32 & -40.1 & -40.1 & -40.1 & -40.1 & -40.1 & -33 & -33 \\
$\lambda_{5}$ & -35 & -41 & -41 & -33 & NA & -37 & -41.1 & -41.1 & -41.1 & -41.1 & -41.1 & -41.1 \\
$\lambda_{6}$ & -35 & -35 & -35 & -35 & -37 & NA & -41 & -41 & -41 & -41 & -41 & -41 \\
$\lambda_{7}$ & -31.1 & -34.8 & -34.6 & -41.1 & -41.7 & -37.1 & NA & -37.3 & -42.2 & -42.4 & -42.6 & -42.8 \\
$\lambda_{8}$ & -34.5 & -34.7 & -34.8 & -41.9 & -41.6 & 41.5 & -37.2 & NA & -37.7 & -41.9 & -41.6 & -41.3 \\
$\lambda_{9}$ & -34.5 & -34.7 & -34.9 & -41.5 & -41.6 & -41.7 & -41.8 & -36.9 & NA & -37.3 & -41.7 & -41.6 \\
$\lambda_{10}$ & -34.7 & -34.7 & -34.6 & -41.5 & -41.6 & -41.6 & -41.6 & -41.6 & -37.2 & NA & -38 & -41.9 \\
$\lambda_{11}$ & -34.8 & -35 & -34 & -41.8 & -41.8 & -41.5 & -41.5 & -41.6 & -41.6 & -37.9 & NA & -38.7 \\
$\lambda_{12}$ & -34 & -34 & -34 & -30 & -30 & -33.4 & -33.4 & -33.4 & -33.4 & -33.3 & -35.8 & NA \\
\hline
\end{tabular}

TABLE 5: Comparison of the proposed and existing DWDM system.

\begin{tabular}{|c|c|c|c|c|c|c|c|c|}
\hline \multirow{2}{*}{$\begin{array}{l}\text { Authors, year, and } \\
\text { reference number }\end{array}$} & \multirow{2}{*}{$\begin{array}{c}\text { Number of } \\
\text { output ports }\end{array}$} & \multicolumn{2}{|c|}{ Coupling efficiency (\%) } & \multirow{2}{*}{$\begin{array}{l}Q \text { factor } \\
\text { Min }\end{array}$} & \multicolumn{2}{|c|}{ Crosstalk (dB) } & \multirow{2}{*}{$\begin{array}{l}\text { Footprint } \\
(\mu \mathrm{m})^{2}\end{array}$} & \multirow{2}{*}{$\begin{array}{c}\text { Spectral } \\
\text { line-width (nm) }\end{array}$} \\
\hline & & Min & $\operatorname{Max}$ & & Min & $\operatorname{Max}$ & & \\
\hline $\begin{array}{l}\text { Rostami et al. } 2010 \\
{[12]}\end{array}$ & 4 & 42.5 & 86.5 & 3006 & -30.00 & -14.2 & 536 & $\begin{array}{c}0.4 \\
\text { NUSL }^{*}\end{array}$ \\
\hline Liu et al. 2012 [13] & 4 & 99 & 99.5 & 90 & - & - & - & $\begin{array}{c}20 \\
\text { NUSL }^{*}\end{array}$ \\
\hline $\begin{array}{l}\text { Alipour-Banaei et } \\
\text { al. } 2013 \text { [18] }\end{array}$ & 4 & 45 & 63 & 561 & -23.70 & -7.5 & 422.4 & $\begin{array}{c}2.8 \\
\text { NUSL }^{*}\end{array}$ \\
\hline $\begin{array}{l}\text { Gupta and Janyani } \\
2014[14]\end{array}$ & 4 & 40 & 80 & 7795 & - & - & - & $\begin{array}{c}0.2 \\
\text { USL }^{*}\end{array}$ \\
\hline Kuo et al. 2007 [15] & 6 & 25 & 60 & 416 & -27 & -17 & - & $\begin{array}{c}3.2 \\
\text { NUSL }^{*}\end{array}$ \\
\hline $\begin{array}{l}\text { Rakhshani et al. } \\
2013 \text { [16] }\end{array}$ & 6 & 81 & 100 & 2319 & -35 & -23 & - & NUSL ${ }^{*}$ \\
\hline Tian et al. 2013 [19] & 8 & 65 & 90 & 1969 & -18 & -15 & 882 & $\begin{array}{c}0.8 \\
\text { NUSL }^{*}\end{array}$ \\
\hline $\begin{array}{l}\text { Mehdizadeh and } \\
\text { Soroosh } 2016 \text { [17] }\end{array}$ & 8 & 94 & 98 & 1723 & -40 & -11.2 & 495 & $\begin{array}{c}1 \\
\text { NUSL }^{*} \\
\end{array}$ \\
\hline Proposed design & 12 & 90 & 99 & 7783.5 & -42 & -30 & 784 & $\begin{array}{c}0.2 \\
U S L^{*}\end{array}$ \\
\hline
\end{tabular}

*USL: uniform spectral linewidth for dropped wavelengths.

NUSL: nonuniform spectral linewidth for dropped wavelengths.

with nonuniform spectral line-width for drop wavelengths is replaced with a uniform spectral line-width one. The functional parameters are significantly enhanced with smaller size and the higher number of channels and hence the proposed demultiplexer can be implemented in the Photonic Integrated Circuits.

\section{Optimization of Rods and Wavelengths in DWDM}

The novel DWDM demultiplexer tunes the rods and wavelength of different sizes and wavelengths. The size of the rods ranging from $41.5 \mathrm{~nm}$ to $46.45 \mathrm{~nm}$ shows the better performance compared to the below $40 \mathrm{~nm}$ rod size. The corresponding wavelength at $1554.5 \mathrm{~nm}$ and below the rod size of $40 \mathrm{~nm}$ shows more distortions. The maximum size of the rod without distortion has been observed at $65 \mathrm{~nm}$. The rods and wavelength are optimized with regression analysis [26, 27] only for 12-channel DWDM. For the proposed 12-channel DWDM demultiplexer, it works better for the Si rods and background air with the refractive indexes of 3.48 and 1.0, respectively, and each rod has a radius $R=110 \mathrm{~nm}$, where lattice constant $a=560 \mathrm{~nm}$ and silicon permittivity $\varepsilon(\mathrm{Si})=$ 11.9. 


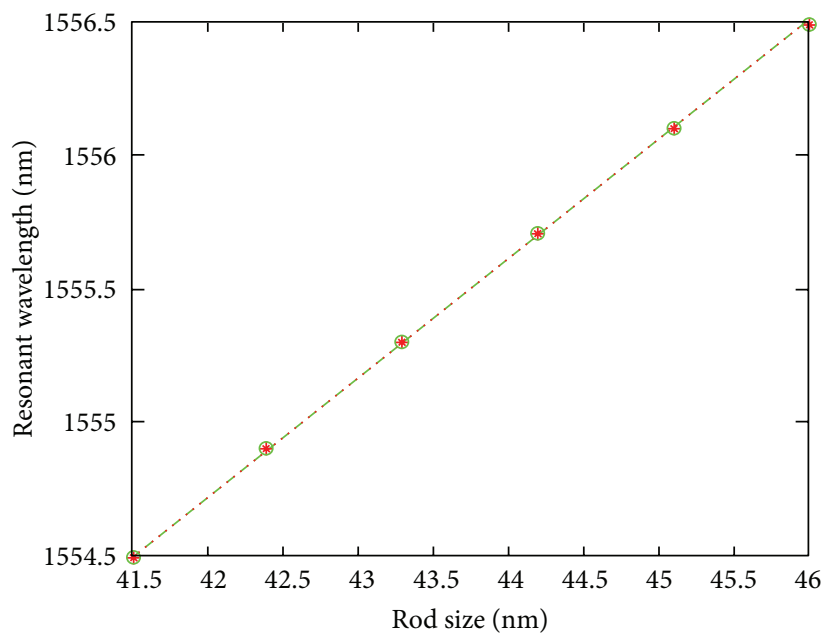

(a)

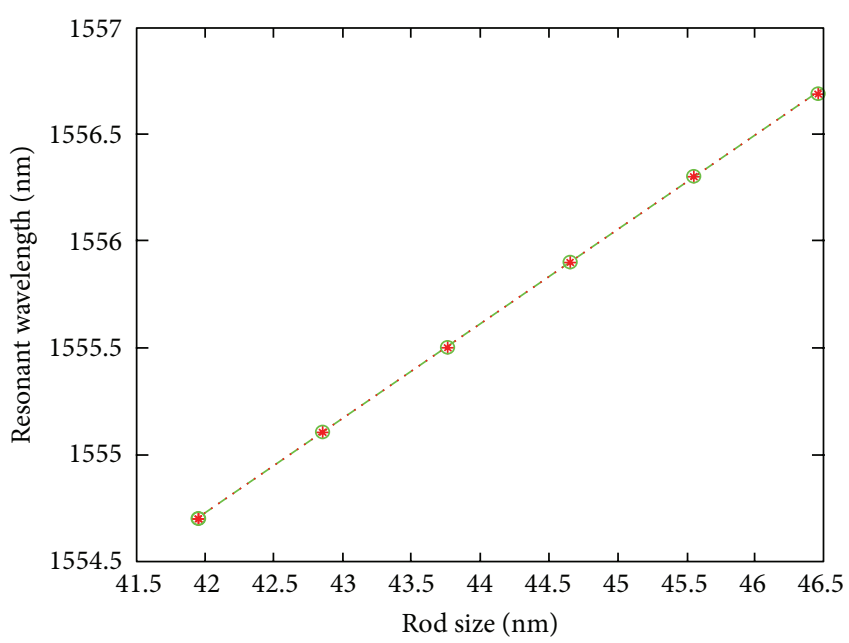

(b)

FIGURE 10: Effect of rods radius with the odd wavelength (a) and even wavelength (b).

The rods of different size $(x)$ taken as $n$ data points with respect to wavelength $(y)$ and data point are $\left\{\left(x_{i}, y_{i}\right), i=\right.$ $1, \ldots, n\}$.

The straight line equation for data points is described with $x$ as rod size and $y$ as wavelength:

$$
y_{i}=\alpha+\beta x_{i}
$$

In (5), $\alpha$ is the interrupt for wavelength and $\beta$ is the slope. We can provide the best fit for rod size and wavelength, the best fit derived with the least square approach, which reduces the sum of square residual of linear regression model.

By solving linear regression equations, we find the value optimization of rods and wavelength:

$$
y=0.444(x)+1533.5
$$

From the above regression model we derived

$$
\begin{aligned}
\text { Wavelength }= & 0.444(\text { rods size }) \\
& + \text { interrupt for wavelength, }
\end{aligned}
$$

where $y$ is the wavelength, $x$ is the different rod size, and $\alpha$ is the interrupt for wavelength.

In the proposed demultiplexer, radius of inner microcavity rod $\left(R_{m}\right)$ is the important parameter for filtering the specific desired wavelengths. To demultiplex the desired wavelength, the radius of the rod $\left(R_{m}\right)$ changes the odd resonant wavelength and the even resonant wavelength changes due to the change of refractive index as shown in Figure 10.

\section{Summary}

The proposed twelve-channel Dense Wavelength Division Multiplexing demultiplexer with the two-dimensional photonic crystal square resonant cavity fulfils the ITU-T recommendation of G.694.1 DWDM systems. The novelty in proposed DWDM system drops the desired wavelength tuned with the radius of the inner $\operatorname{rod}\left(R_{m}\right)$, which is positioned in the microcavity. The spectral response of the proposed DWDM performs with an average of about $96 \%$ of transmission efficiency, $-42 \mathrm{~dB}$ crosstalk with the quality factor of 8000 . Further, the channel spacing and spectral line-width between the channels are $0.2 \mathrm{~nm} / 25 \mathrm{GHz}$ and $0.2 \mathrm{~nm}$, respectively. The proposed PC based demultiplexer is excellent in fulfilling the requirements of ITU-T G.694.1 DWDM system and size is very small, about $784 \mu \mathrm{m}^{2}$; it could be integrated for the integrated optics. From the results, the existing DWDM system with nonuniform spectral line-width for drop wavelengths is replaced with a uniform spectral linewidth one. The crosstalk of proposed DWDM demultiplexer improves about $-42 \mathrm{~dB}$ compared to previous works.

\section{Competing Interests}

There are no competing interests regarding the publication of this paper.

\section{Acknowledgments}

The authors are very much grateful to Dr. B. Chidambararajan, Principal of Valliammai Engineering College, Kattankulathur, and Dr. R. Nakkeeran, Head of Electronics Engineering Department, School of Engineering \& Technology, Pondicherry University, for their valid suggestions and inputs at suitable levels. Authors are extremely thankful to Professor N. Lakshmana Perumal, Professor and Head of English Department of Valliammai Engineering College, Kattankulathur, for his help in correcting the grammatical errors in the paper. Venkatachalam Rajarajan Balaji would like to acknowledge Dr. N. R. Shanker, Manager, Research and Development, Chase Technologies, Chennai, for his support at different times. 


\section{References}

[1] B. Hoanca, "DWDM fundamentals, components, and applications," Journal of Optical Networking, vol. 1, no. 5, pp. 184-185, 2002.

[2] A. K. Dutta, N. K. Dutta, and M. Fujiwara, Eds., WDM Technologies: Passive Optical Components: Passive Optical Components, Academic Press, 2003.

[3] I. Nishi, T. Oguchi, and K. Kato, "Broad-passband-width optical filter for multi/demultiplexer using a diffraction grating and a retroreflector prism," Electronics Letters, vol. 21, no. 10, pp. 423424, 1985.

[4] X. Xing and Y. Wang, "A planar lightwave circuit based on optical add-drop multiplexer with 16 tunable wavelength channels," Advanced Materials Research, vol. 216, pp. 661-665, 2011.

[5] E. Yablonovitch, "Inhibited spontaneous emission in solid-state physics and electronics," Physical Review Letters, vol. 58, no. 20, pp. 2059-2062, 1987.

[6] J. D. Joannopoulos, P. R. Villeneuve, and S. Fan, "Photonic crystals: putting a new twist on light," Nature, vol. 386, no. 6621, pp. 143-149, 1997.

[7] K. B. Chung and S. W. Hong, "Wavelength demultiplexers based on the superprism phenomena in photonic crystals," Applied Physics Letters, vol. 81, no. 9, pp. 1549-1551, 2002.

[8] M. Bayindir, B. Temelkuran, and E. Ozbay, "Photonic-crystalbased beam splitters," Applied Physics Letters, vol. 77, article 3902, 2000.

[9] H. Alipour-Banaei, M. G. Rabati, P. Abdollahzadeh-Badelbou, and F. Mehdizadeh, "Application of self-collimated beams to realization of all optical photonic crystal encoder," Physica E: Low-Dimensional Systems and Nanostructures, vol. 75, pp. 7785, 2016.

[10] R. Savarimuthu and N. Rangaswamy, "Coupled mode theory analysis for circular photonic crystal ring resonator-based adddrop filter," Optical Engineering, vol. 51, no. 11, Article ID 114001, 2012.

[11] K. V. Shanthi and S. Robinson, "Two-dimensional photonic crystal based sensor for pressure sensing," Photonic Sensors, vol. 4, no. 3, pp. 248-253, 2014.

[12] A. Rostami, F. Nazari, H. A. Banaei, and A. Bahrami, "A novel proposal for DWDM demultiplexer design using modifiedT photonic crystal structure," Photonics and NanostructuresFundamentals and Applications, vol. 8, no. 1, pp. 14-22, 2010.

[13] W. Liu, D. Yang, H. Tian, and Y. Ji, "Optimization transmission of photonic crystal coupled cavity and design of demultiplexer for wavelength division multiplexing application," Optical Engineering, vol. 51, no. 8, Article ID 084002, 2012.

[14] N. D. Gupta and V. Janyani, "Dense wavelength division demultiplexing using photonic crystal waveguides based on cavity resonance," Optik-International Journal for Light and Electron Optics, vol. 125, no. 19, pp. 5833-5836, 2014.

[15] C.-W. Kuo, C.-F. Chang, M.-H. Chen, S.-Y. Chen, and Y.-D. Wu, "A new approach of planar multi-channel wavelength division multiplexing system using asymmetric super-cell photonic crystal structures," Optics Express, vol. 15, no. 1, pp. 198-206, 2007.

[16] M. R. Rakhshani, M. A. Mansouri-Birjandi, and Z. Rashki, "Design of six channel demultiplexer by heterostructure photonic crystal resonant cavity," International Research Journal of Applied and Basic Sciences, vol. 4, no. 4, pp. 976-984, 2013.
[17] F. Mehdizadeh and M. Soroosh, "A new proposal for eightchannel optical demultiplexer based on photonic crystal resonant cavities," Photonic Network Communications, vol. 31, no. 1, pp. 65-70, 2016.

[18] H. Alipour-Banaei, F. Mehdizadeh, and S. Serajmohammadi, "A novel 4-channel demultiplexer based on photonic crystal ringresonators," Optik, vol. 124, no. 23, pp. 5964-5967, 2013.

[19] H. Tian, G. Shen, W. Liu, and Y. Ji, "Integration of both dense wavelength-division multiplexing and coarse wavelengthdivision multiplexing demultiplexer on one photonic crystal chip," Optical Engineering, vol. 52, no. 7, Article ID 076110, 2013.

[20] V. R. Balaji, M. Murugan, and S. Robinson, "DWDM demultiplexer using inverted-p photonic crystal structure," International Journal of Applied Engineering Research (IJAER), vol. 10, no. 6, pp. 5688-5692, 2015.

[21] J. Clerk Maxwell, "A dynamical theory of the electromagnetic field," Journal of Philosophical Transactions of the Royal Society of London, vol. 155, no. 1865, pp. 459-512, 2015.

[22] K. S. Kunz and R. J. Luebbers, The Finite Difference Time Domain Method for Electromagnetic, CRC Press, New York, NY, USA, 1993.

[23] C.-P. Yu and H.-C. Chang, "Yee-mesh-based finite difference eigenmode solver with PML absorbing boundary conditions for optical waveguides and photonic crystal fibers," Optics Express, vol. 12, no. 25, pp. 6165-6177, 2004.

[24] J. Lutkenhaus, D. George, D. Lowell, B. Arigong, H. Zhang, and Y. Lin, "Registering functional defects into periodic holographic structures," Applied Optics, vol. 54, no. 23, pp. 7007-7012, 2015.

[25] K. N. Sediq, D. Coles, P. W. Fry, and D. G. Lidzey, "Plasmonic gold nanodiscs fabricated into a photonic-crystal nanocavity," Nanotechnology, vol. 27, no. 22, Article ID 225203, 2016.

[26] H. Alipour-Banaei and F. Mehdizadeh, "Bandgap calculation of $2 \mathrm{D}$ hexagonal photonic crystal structures based on regression analysis," Journal of Optical Communications, vol. 34, no. 4, pp. 285-293, 2013.

[27] N. R. Draper, H. Smith, and E. Pownell, Applied Regression Analysis, vol. 3, Wiley, New York, NY, USA, 1966. 

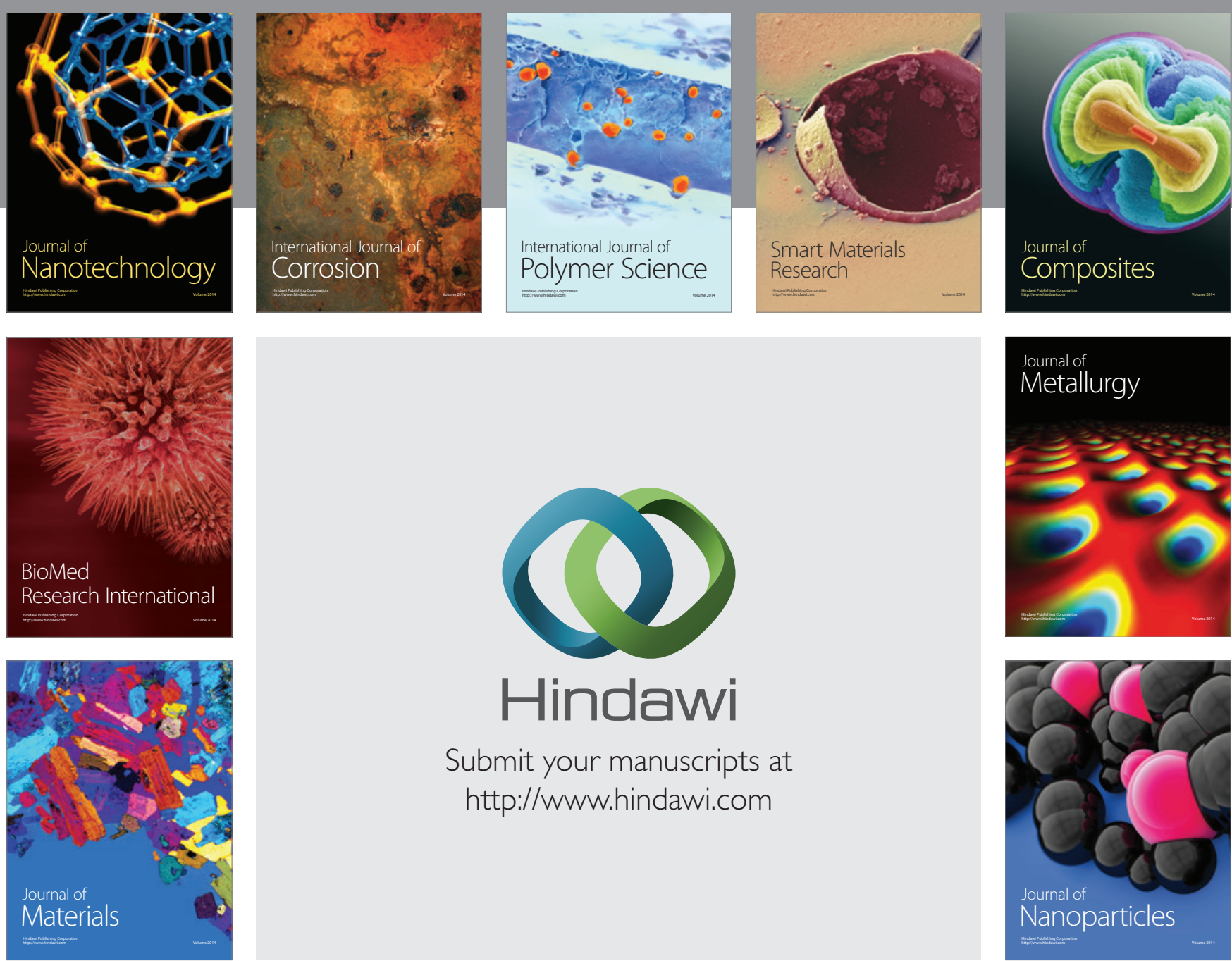

\section{Hindawi}

Submit your manuscripts at

http://www.hindawi.com

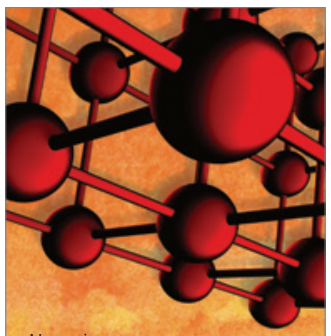

Materials Science and Engineering
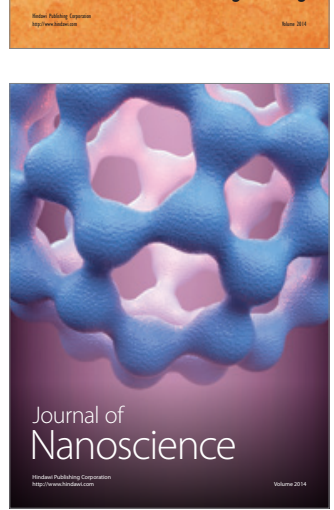
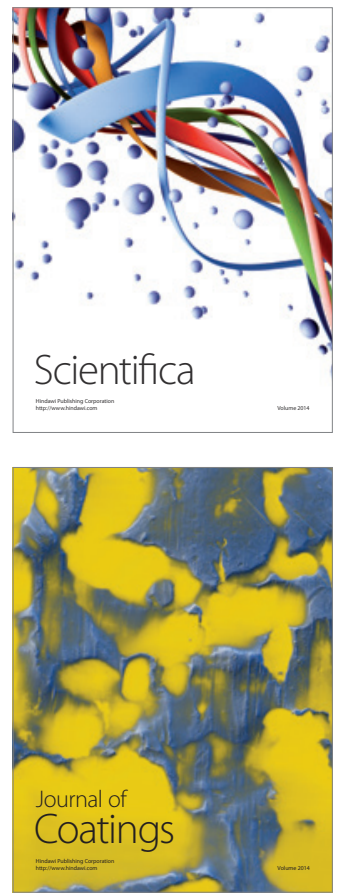
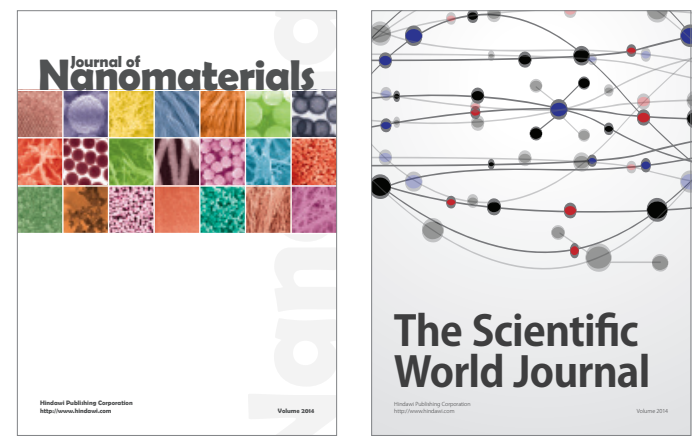

The Scientific World Journal
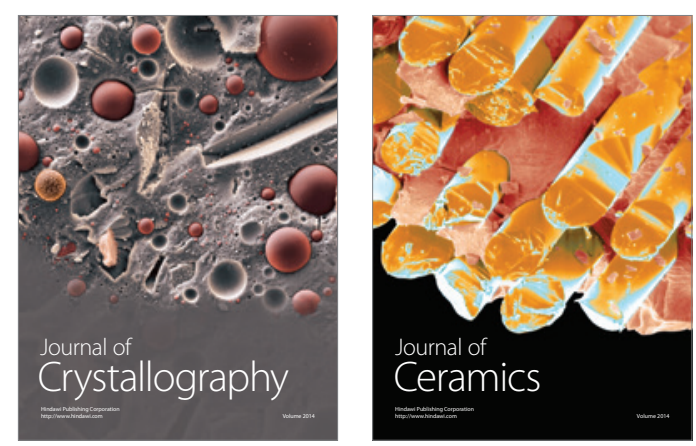
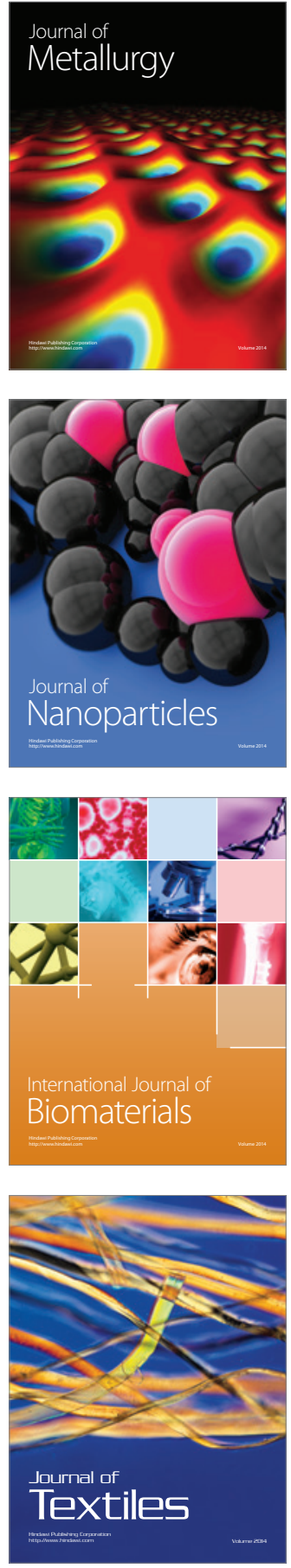